\title{
Turbulent Kinetic Energy in Normal and Myopathic Left Ventricles
}

\author{
Jacub Zajac, Jonatan Eriksson, Petter Dyverfeldt, Ann F. Bolger, \\ Tino Ebbers and Carl-Johan Carlhäll
}

\section{Linköping University Post Print}

\section{Tweet}

N.B.: When citing this work, cite the original article.

Original Publication:

Jacub Zajac, Jonatan Eriksson, Petter Dyverfeldt, Ann F. Bolger, Tino Ebbers and Carl-Johan Carlhäll, Turbulent Kinetic Energy in Normal and Myopathic Left Ventricles, 2015, Journal of Magnetic Resonance Imaging, (41), 4, 1021-1029.

http://dx.doi.org/10.1002/jmri.24633

Copyright: Wiley http://eu.wiley.com/WileyCDA/

Postprint available at: Linköping University Electronic Press http://urn.kb.se/resolve?urn=urn:nbn:se:liu:diva-99957 


\section{Turbulent Kinetic Energy in Normal and Myopathic Left Ventricles}

Jakub Zajac ${ }^{1,2,3}$ (MD), Jonatan Eriksson ${ }^{1,2}(\mathrm{MS})$, Petter Dyverfeldt ${ }^{1,2}(\mathrm{PhD})$, Ann F. Bolger ${ }^{1,4}$ (MD), Tino Ebbers ${ }^{1,2,5}(\mathrm{PhD})$, Carl-Johan Carlhäl1 ${ }^{1,2,3}(\mathrm{MD}, \mathrm{PhD})$

${ }^{1}$ Division of Cardiovascular Medicine, Department of Medical and Health Sciences, Linköping University, Linköping, Sweden

${ }^{2}$ Center for Medical Image Science and Visualization (CMIV), Linköping University, Linköping, Sweden

${ }^{3}$ Department of Clinical Physiology, UHL, County Council of Östergötland, Linköping, Sweden

${ }^{4}$ Department of Medicine, University of California San Francisco, San Francisco, CA, USA

${ }^{5}$ Division of Media and Information Technology, Department of Science and Technology /

Swedish e-Science Research Centre (SeRC), Linköping University, Linköping, Sweden

Correspondence: Jakub Zajac, MD

Department of Medical and Health Sciences

Division of Cardiovascular Medicine, Linköping University

SE-581 85 Linköping

E-mail: jakubzajac@gmail.com

Telephone: +46 101030000

Fax: +46(0)13145949

This study was funded by the Swedish Heart-Lung foundation, the Swedish Research Council and the European Research Council.

Running Title: Turbulent Flow in Normal and Myopathic Hearts 


\begin{abstract}
Purpose: To assess turbulent kinetic energy (TKE) within the left ventricle (LV) of healthy subjects using novel 4D flow MRI methods and to compare TKE values to those from a limited group of patients with a spectrum of dilated cardiomyopathy (DCM).

Methods: 4D flow and morphological MRI-data were acquired in 11 healthy subjects and 9 patients with different degrees of diastolic dysfunction. TKELV was calculated within the LV at each diastolic time frame. At peak early (E) and late (A) diastolic filling, the TKE $E_{L V}$ was compared to transmitral peak velocity, LV diameter and mitral annular diameter.

Results: In the majority of subjects, TKELv peaks were observed at E and A. Peak TKELV at E was not different between the groups ( $\mathrm{p}=0.33$ ), and correlated with mitral annular dimensions $\left(\mathrm{r}^{2}=0.32, \mathrm{p}=0.01\right)$. Peak TKE $\mathrm{LV}$ at $\mathrm{A}$ was higher in DCM patients compared to healthy subjects $(3.0 \pm 1.8$ vs $1.5 \pm 0.8 \mathrm{~mJ}, \mathrm{p}=0.02)$, and correlated with LV diameter and transmitral velocity $\left(\mathrm{r}^{2}=0.36, \mathrm{p}=0.01\right.$ and $\mathrm{r}^{2}=0.47, \mathrm{p}<0.01$, respectively $)$.
\end{abstract}

Conclusions: In LVs of healthy subjects, TKE values are low. Values are highest during early diastole, and diminish with increasing LV size. In a heterogeneous group of DCM patients, late diastolic TKE values are higher than in healthy subjects.

Key Words: magnetic resonance imaging, blood flow, turbulent flow, cardiac function, diastolic dysfunction, heart failure 


\section{INTRODUCTION}

In normal resting adults the relatively low left ventricular (LV) inflow and outflow velocities as well as paucity of the third and fourth (gallop) heart sounds and murmurs have pointed to the presumption of fairly small amounts of turbulent blood flow within the normal LV $(1,2)$. In heart failure patients the gallop sounds and murmurs are more prevalent. The third and fourth heart sounds are thought to coincide with deceleration of blood flowing into the LV at early and late diastolic filling, respectively $(3,4)$. Decelerating blood flow is known to have a destabilizing effect on the flow resulting in disturbed and transitionally turbulent flow $(1,5)$.

Earlier studies based on ultrasound (6,7) and computational fluid dynamics (8) as well as magnetic resonance imaging (MRI) (9) have proposed that the blood flow patterns observed within normal LVs are altered in LVs of heart failure patients. Furthermore, previous studies with three-directional three-dimensional cine phase-contrast MRI (4D flow MRI) have shown that a substantial portion of the LV inflow passes directly to outflow in normal LVs (10). In contrast, in LVs of heart failure patients a substantial portion of the inflow is retained within the LV, and this altered flow pattern is associated with decreased preservation of LV inflow kinetic energy $(10,11)$.

Previous 4D flow studies of the LV have been based on mean velocity data and have thus been unable to assess disturbances due to transitional and turbulent flow as characterized by rapid and apparently random velocity fluctuations $(10,11)$. When blood flow becomes transitionally turbulent, turbulent kinetic energy (TKE) is transferred from the mean flow to small turbulent eddies where viscous forces dominate and the energy is dissipated into heat. As all TKE is dissipated, the TKE can be seen as a measure of flow inefficiency. Recent developments in 4D flow MRI permit non-invasive estimation of TKE (12-14). This has been 
used to assess TKE in normal aortas $(15,16)$, the setting of vascular $(12)$ and valvular stenoses (15) as well as in the left atrium (13). The low values of TKE measured with 4D flow MRI in normal aortas agree well with early hot-wire anemometry studies (15-17). However, the amount, timing and regional distribution of intraventricular TKE in healthy left ventricles has not been explored. On the basis of the Reynolds number, which is widely used to define critical values for the transition from laminar to turbulent flow $(5,18)$, albeit in non-pulsatile pipe flow, one might expect that larger LV size would promote higher TKE values and thus lower flow efficiency. Patients with dilated cardiomyopathy and LV systolic dysfunction with varying degrees of demonstrable diastolic dysfunction could be expected to demonstrate differences in TKE from normal under conditions of larger LV volumes and depressed contractility.

The purpose of this study is to assess TKE in the left ventricles of healthy subjects and to compare those findings to results in a spectrum of patients with DCM. We hypothesized that the TKE of diastolic inflow would be larger in the dilated and hypocontractile LVs of heart failure patients compared to normal LVs. 


\section{MATERIALS AND METHODS}

\section{Study Population}

Eleven healthy subjects and ten patients with dilated cardiomyopathy (DCM) with variable degrees of diastolic dysfunction were enrolled in the study (Table 1). All healthy subjects had normal electrocardiographic and echocardiographic examinations. Exclusion criteria for (I) all subjects: contraindication to MRI examination; (II) healthy subjects: a history of prior or current heart disease or the use of cardiac medication; and (III) DCM patients: significantly irregular cardiac rhythm, more than mild valvular disease, less than mild LV systolic dysfunction, less than mild LV dilatation. All subjects were in sinus rhythm; all patients were clinically compensated and euvolemic. One patient was excluded during the data analysis process due to technical circumstances. The study was approved by the regional Ethical Review Board and all subjects gave written informed consent before participation.

\section{Data Acquisition And Processing}

A clinical 1.5T MR scanner (Philips Achieva; Philips Medical Systems, Best, the Netherlands) with a five element cardiac SENSE coil was used to acquire 4D flow MRI data and cine bSSFP data of the LV in all subjects.

The 4D flow MRI data for the assessment of velocity and TKE were acquired during freebreathing using a retrospectively navigator-gated gradient-echo pulse-sequence with asymmetric three-directional motion-encoding and retrospective, vector cardiogram controlled cardiac gating (12,19). Acquisition parameters included a VENC of $100 \mathrm{~cm} / \mathrm{s}$, a TR/TE of 6.3/3.7 ms, and a flip angle of 8 degrees, parallel imaging by sensitivity encoding (SENSE) with a speed-up factor of 2, and a k-space segmentation factor of two. The spatial resolution was $3 \times 3 \times 3 \mathrm{~mm}^{3}$ and the field-of-view was adjusted for each subject to cover the 
left heart. Velocity fields were reconstructed on the scanner using standard phase-difference algorithms and were corrected offline for background phase offsets and velocity aliasing. The TKE per unit volume is defined as (20):

$\mathrm{TKE}=\frac{1}{2} \rho \sum_{i=1}^{3} \sigma_{i}^{2}\left[\mathrm{~J} \mathrm{~m}^{-3}\right] \quad[$ Eq. 1]

where $\rho$ is the fluid density and $\sigma_{i}$ is the velocity fluctuation intensity in three mutually perpendicular directions $i$. Three-directional PC-MRI with asymmetric four-point motion encoding, as used here, provides the three necassary $\sigma_{i}$ and thus enables TKE estimation (12). The relationship used to compute $\sigma_{i}$ appears as $(12,14)$ :

$\sigma_{i}=\frac{1}{k_{\mathrm{v}}} \sqrt{2\left(\frac{|S|}{\left|S_{i}\right|}\right)}$

where $|S|$ and $\left|S_{i}\right|$ denote the amplitude of a complex-valued MR signal acquired with zero first-order motion sensitivity and motion sensitivity in direction $i$, respectively. $|S|$ and $\left|S_{i}\right|$ were obtained by reconstructing the magnitude images of the four individual flow-encoding segments. $k_{\mathrm{v}}(=\pi / \mathrm{VENC})$ describes the net motion sensitivity. The fluid density was assumed to be $1.060 \mathrm{~kg} / \mathrm{m}^{3}$.

Cine bSSFP imaging during end-expiratory breath holds was used to acquire short-axis and two, three- and four-chamber long-axis images in 30 time frames across the cardiac cycle. A slice thickness of $8 \mathrm{~mm}$ was used and the acquired pixel size was $2.19 \times 1.78 \mathrm{~mm}^{2}$ and $1.67 \mathrm{x}$ $1.78 \mathrm{~mm}^{2}$ for the short- and long-axis images, respectively. Reconstructed pixel size was 1.25 $\mathrm{mm}^{2}$ for the long-axis images and $1.37 \mathrm{~mm}^{2}$ for the short-axis images. The bSSFP data were used for segmentation of ventricular volumes.

A standard echocardiographic examination was performed using a Vivid 7 scanner and a 2.0 MHz probe (GE, Vingmed Ultrasound, Horten, Norway). The echo Doppler data were used to 
evaluate enrollment criteria and for characterization of LV diastolic function at rest according to mitral and pulmonary venous inflow and mitral annular velocities.

\section{Data Analysis}

The LV was segmented at each diastolic time frame from the short-axis image stack using free software Segment (v1.9R2046, Medviso, Lund, Sweden) (21). The end systolic and the end diastolic time frames were defined using visual inspection of a 3-chamber image as the first time frame after aortic valve closure and the first time frame after mitral valve closure, respectively. The segmentation was done with identification of the subendocardium of the compact ventricular myocardium in order to certify coverage of entire left ventricular volume. Preliminary investigations showed that the inclusion of myocardium had negligible effect on the sum of TKE.

The 3D velocity field and TKE within the segmented LV were visualized in all subjects throughout the cardiac cycle using the commercial visualization software EnSight, (EnSight, CEI Inc, Research Triangle Park, NC, USA). By visual inspection of a 3D volume rendering of the intraventricular TKE, the spatial distribution of elevated TKE was graded according to seven different LV regions: the basal third of the LV was divided into an anteroseptal region (outflow tract), a central (submitral) region and an inferolateral region; the midventricular third of the LV was divided into an anteroseptal region, a central region and an inferolateral region; the apical third of the LV was not divided into any further regions. Two investigators (JZ and CJC, 4 and 10 years of experience in 4D flow MRI, respectively) performed the visual inspection independently, and subsequently reached consensus on the spatial distribution of elevated TKE within the LV during diastole. The TKE within the segmented 
LV was integrated to obtain the TKE within the entire LV (TKELV) at each diastolic time frame. Further, the sum of TKELV over all diastolic time frames was computed.

The highest values of $\mathrm{TKE}_{\mathrm{LV}}$ (peak $\mathrm{TKE}_{\mathrm{LV}}$ ) during early and late diastolic filling, respectively, were compared for all normal and DCM subjects. During early diastolic filling peak $\mathrm{TKE}_{\mathrm{LV}}$ was compared to measures of peak transmitral velocity during early filling, as well as to anteroseptal-posterolateral dimensions of the LV and the mitral annulus at this time point. The corresponding comparisons were also performed during late diastolic filling. The peak TKELV values during early and late filling, respectively, were chosen as markers for comparison, as these values are readily identified in the data sets and because early and late filling are the main events of the diastolic phase. In order to minimize the probability that spurious values in single voxels would contaminate the TKELV values, the following controlprocedure was performed in all subjects: the TKELv over diastole curve was inspected, and then compared to the spatial distribution of elevated TKE within the segmented LV during diastole obtained by visual inspection of a 3D volume rendering of the TKE. The peak transmitral velocity values were controlled for in a similar way in all subjects: based on the 3D velocity field, the spatial localization of the peak transmitral velocity values during early and late filling, respectively, were identified. In all subjects this site was very close to the estimated position of the vena contracta.

Subvoxel variations in mean velocity due to shear, partial volume or opposing flow effects can theoretically affect MR TKE estimation (22). The impact of this effect was estimated in each subject based on methods described previously (22). In brief, the subvoxel mean velocity variation was modeled as a uniform distribution (linear velocity gradient) and its contribution to the actual intravoxel velocity distribution was estimated by convolution. The subvoxel 
mean velocity variations were estimated by differentiation of the measured 3D velocity field in each time frame.

\section{Statistical Evaluation}

All values are given as group mean \pm 1 SD unless otherwise specified. All quantitative parameters showed a normal distribution in the data according to the Kolmogorov-Smirnov test. TKE $\mathrm{LV}$ at early and late diastolic filling and the sum of TKE $\mathrm{LV}_{\mathrm{L}}$ from all diastolic time frames were compared between DCM and healthy subjects using a Student's t-test for unpaired observations. Pearson's correlations were calculated between peak TKELV and LV diameter, mitral annular diameter, and transmitral peak velocity during early and late diastolic filling, respectively. The statistical significance was set to $\mathrm{P}<0.05$. The software used for the statistical analyses was Statistica 9.1 (StatSoft). 


\section{RESULTS}

There was no significant difference in age $(51 \pm 13$ vs $43 \pm 18, \mathrm{p}=0.30)$ or heart rate $(62 \pm 12 \mathrm{vs}$ $71 \pm 10, \mathrm{p}=0.08$ ) between DCM patients and healthy subjects. The LV end-diastolic volume and LV ejection fraction were higher and lower, respectively, in DCM patients compared to healthy subjects $(173 \pm 33$ vs $144 \pm 25$, and $41 \pm 5$ vs $62 \pm 3 \%$, both $p<0.05)$. At rest, 5 of 9 patients had diastolic dysfunction of different severities, while all healthy subjects showed normal diastolic function (Table 1). None of the subjects had mitral or aortic valve disease.

By visual inspection, in LVs of all healthy subjects, regions of elevated TKE were observed mainly following the early filling velocity peak, and were located predominantly in the submitral region in the basal third of the LV (Figure 1; Supplemental Movie S1). In the majority of these healthy subjects, additional regions of elevated TKE were observed near the anteroseptal portion of the inflow ring vortex with extension into the outflow tract, especially following early filling. Quantitatively, in healthy subjects, TKELV values were highest during early diastole and diminished as LV volume increased throughout diastole (Figure 2).

In contrast to the normal subjects, regionally elevated TKE was visible following both early and late filling in DCM LVs, with the exception of one of the patients with restrictive filling patterns where no regional increase in TKE was seen during late filling. Compared to normals, in the myopathic LVs the spatial distribution of elevated TKE extended further into the inferolateral and central LV, reaching the apical half of the chamber in the majority of subjects (Figure 3; Supplemental Movies S2 and S3). Quantitatively, compared to healthy subjects, there was a trend for TKE $\mathrm{LV}$ values to increase as the LV size increased in the majority of the DCM patients (Figure 4). 
In the majority of all subjects, peak values of TKE $\mathrm{LV}$ were obtained at early and late diastolic filling. The time point of peak TKELV occurred approximately $25-75 \mathrm{~ms}$ later than the time point of the transmitral peak velocity, at both early and late diastolic filling. Peak TKELV at late filling was higher in DCM compared to healthy subjects $(3.0 \pm 1.8$ vs $1.5 \pm 0.8 \mathrm{~mJ}, \mathrm{p}=0.02)$; there was no difference in peak $\mathrm{TKE}_{\mathrm{LV}}$ at early filling between the groups $(3.0 \pm 1.0$ vs $2.5 \pm 1.2$ $\mathrm{mJ}, \mathrm{p}=0.33$ ) (Figure 5). The sum of $\mathrm{TKE}_{\mathrm{LV}}$ over all diastolic time frames tended to be higher in DCM compared to healthy subjects ( $43 \pm 18$ vs $28 \pm 17 \mathrm{~mJ}, \mathrm{p}=0.09$ ).

LV diameter at early and late diastolic filling was higher in DCM compared to Healthy (5.4 \pm 0.8 vs $3.7 \pm 0.4 \mathrm{~cm}$, and $6.0 \pm 0.6$ vs $4.3 \pm 0.5$, respectively, both $\mathrm{p}<0.001$ ) (Table 2 ). At early and late diastolic filling, respectively, there were no differences in mitral annular dimension ( $3.3 \pm 0.6$ vs $3.1 \pm 0.3 \mathrm{~cm}, \mathrm{p}=0.35$, and $3.3 \pm 0.7$ vs $2.9 \pm 0.3, \mathrm{p}=0.09$, respectively) or transmitral peak velocity $(0.81 \pm 0.1$ vs $0.94 \pm 0.2 \mathrm{~m} / \mathrm{s}, \mathrm{p}=0.09$, and $0.71 \pm 0.3$ vs $0.61 \pm 0.1$, $\mathrm{p}=0.31$, respectively) between the groups (Table 2). At early filling, peak TKELV for both normal and DCM LVs correlated moderately to mitral annular dimension (Figure 6); there

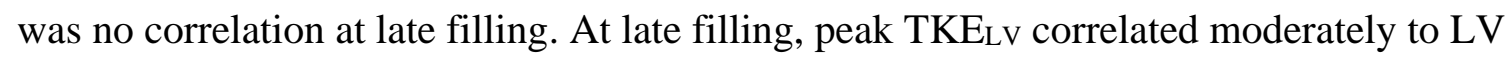
dimension and transmitral peak velocity (Figure 6); there was no correlation at early filling.

Subvoxel variations in mean velocity were found to contribute to $5 \pm 4 \%$ of the measured TKE in healthy subjects and $2 \pm 1 \%$ in DCM patients. 


\section{DISCUSSION}

Although intracardiac flow physiology has been explored for many decades there is still no consensus on whether or not there is significant turbulence within the normal heart. To address this familiar question, we utilized novel 4D flow MRI methods to assess turbulent kinetic energy (TKE) within the LVs of healthy subjects and also to compare normal TKE values, timing and distribution to a spectrum of patients with dilated cardiomyopathy.

The magnitude of TKELV in the healthy subjects ranged from 0 to approximately $5 \mathrm{~mJ}$. This is similar to corresponding TKE values previously reported for the normal ascending aorta $(15,16)$ and left atrium (13). As hypothesized, the TKE values were low in normal left ventricles in comparison to other clinical scenarios where turbulence is present, such as valvular stenosis or regurgitation where values may be up to 10 times as high $(13,15)$.

The TKE in the normal ventricles was observed in the basal third of the LV. The time periods with highest inflow velocity, peak $\mathrm{E}$ and peak $\mathrm{A}$, were associated with increases in the total TKE measured throughout the LV chamber $\left(T_{K E} L V\right)$. As the LV volume increased throughout diastole, there was a decrease in the amount of TKE.

These findings in the healthy subjects were compared to a group of clinically compensated DCM patients who had varying degrees of diastolic dysfunction, from none to severe. The sum of TKELv over all diastolic time frames tended to be higher in the DCM patients; the early diastolic TKE did not differ between normal and DCM patients, but late diastolic TKE values were higher in the myopathic hearts. 
The trend in TKE over the range of LV size resulting from diastolic filling was different between the normal and DCM subjects, raising the question of what might correlate with TKE overall, and what the contributing factors and the consequences of the elevated late diastolic TKE might be.

By combining all normal and patient values together, contributors to TKE could be considered. Disturbed flow might be anticipated in the setting of high inflow velocity (due to increased propensity for transitional and turbulent flow) and/or greater LV size, as suggested by the variables within the formula for the Reynolds number (18).

In the range of early mitral inflow velocities in this combined group, there was no correlation of TKE with velocity. In late diastole, velocity did correlate with inflow velocity, driven by the values in the DCM patients. Even though no significant intergroup difference was observed in transmitral peak velocity at either early or late filling, there was a trend for higher velocities in healthy subjects compared to DCM patients during early filling. This could have contributed to some TKE in healthy subjects at early diastolic filling and thus reduced the difference in TKE between the groups. Another reason for increased TKE only during late filling in DCM may be that the inflowing blood encounters LV pressure which is higher or rises more rapidly during late diastole compared to healthy. In addition, vortices in the basal LV differ between DCM and healthy hearts. Abnormal location or rotational velocities of the vortices might influence the development of TKE.

The LV diameter was larger in DCM patients compared to healthy subjects during both early and late diastolic filling, however TKE was only higher in DCM compared to healthy during late filling. The correlation of TKE with larger intracavitary dimensions at end diastole seen 
in the DCM patients may reflect an increased propensity to transitional and turbulent flow according to the Reynolds number (18).

What might be the impact of the elevated TKE in DCM patients? Previous 4D flow MRI studies have shown that in DCM LVs the majority of the late diastolic inflow volume is retained rather than ejected. This retained LV inflow progresses farther into the LV cavity than in the normal heart and augments the vortex in the center of the LV which begins to build up during early diastolic filling $(11,23)$. In contrast, in normal LVs most of the late diastolic inflow volume passes directly to ensuing ejection (10). These flow patterns are in line with the visual patterns of TKE in the majority of the LVs: in DCM LVs, and in particular at late filling compared to normal LVs, TKE is observed deeper in the central part of the cavity. In contrast, in many of the normal LVs, TKE is seen in the vicinity of the anteroseptal part of the mitral inflow ring vortex, extending into the outflow tract, especially following early filling.

Increased TKE associated with re-circulating blood in the central part of the LV observed in DCM may result in irreversible conversion of kinetic energy into heat by viscous dissipation. The effect on the total cardiac workload is most likely small, but these losses can be a sign of inefficient intra-ventricular flow patterns, in contrast to the flow patterns seen in normal LVs that may favor efficient systolic ejection (10).

In conclusion, TKE values measured with MRI are low in the normal left ventricle, and regionally distributed near the basal LV regions. TKE values in healthy subjects are highest during early diastole, and diminish as the LV continues to fill. In contrast, in clinically compensated DCM patients with a spectrum of diastolic dysfunction severity, by late diastole 
LV TKE values exceed those in normal LVs. Kinetic energy loss due to this relative increase in late diastolic TKE may reflect inefficient flow in dilated and dysfunctional LVs of heart failure patients, correlate with abnormal 4D ventricular flow abnormalities, and possibly be a marker of diastolic dysfunction which is not fully represented by conventional echo Doppler assessments. 


\section{LIMITATIONS}

Variations in the mean velocity within a voxel will theoretically contribute to the intravoxel distribution of spin velocities and thereby result in falsely elevated TKE. Such contributions may have resulted in TKE values that are slightly overestimated. However, as the estimated impact of these contributions was estimated to be less than approximately $5 \%$ of the measured TKE we believe that this effect is negligible. Smaller voxels may be expected to reduce this effect even further. Similarly, a recent study performed an extensive comparison between MRI and particle tracking velocimetry and found that the TKE measured with the two approaches was strongly correlated with a gold-standard particle image velocimetry method across a wide range of TKE values (24).

A limitation of 4D flow MRI is that the acquisition of a dataset takes several hundred cardiac cycles. Variations in cardiac frequency can cause image artifacts. With retrospective cardiac gating, as used here, variations in cardiac frequency are accounted for by individually stretching each RR-interval in the image reconstruction process. In the present study, the stretching was done in a nonlinear manner so as to account for the fact that the duration of systole is almost constant. Additionally, gating errors and arrhythmias were accounted for by rejecting RR-intervals shorter than $90 \%$ or longer than $120 \%$ of the average duration of the eight preceding RR-intervals. Rejected RR-intervals were re-acquired in the subsequent heartbeat.

The current data relate to a limited number of subjects in the supine position at rest and in sinus rhythm. One factor that limits the use of larger cohorts is the long acquisition time of 4D flow MRI. The 1.5T 4D flow MRI protocol used in the present study resulted in nominal scan times of 10-15 minutes and respiratory navigator efficiencies of about 50\%. Ongoing efforts 
are directed at reducing the scan time (25). Another time-consuming aspect of the present study was the time-resolved segmentation of the LV based on short-axis bSSFP images. Automated segmentation would facilitate application in a larger number of subjects. 


\section{REFERENCES}

1. Sabbah HN, Stein PD. Turbulent blood flow in humans: its primary role in the production of ejection murmurs. Circ Res 1976;38:513-525.

2. Murgo JP. Systolic ejection murmurs in the era of modern cardiology: what do we really know? J Am Coll Cardiol 1998;32:1596-1602.

3. Kono T, Rosman H, Alam M, Stein PD, Sabbah HN. Hemodynamic correlates of the third heart sound during the evolution of chronic heart failure. J Am Coll Cardiol 1993;21:419-423.

4. McGuire AM, Hagley MT, Hall AF, Kovács SJ. Relationship of the fourth heart sound to atrial systolic transmitral flow deceleration. Am J Physiol 1997;272:H1527-1536.

5. Yoganathan AP, Cape EG, Sung HW, Williams FP, Jimoh A. Review of hydrodynamic principles for the cardiologist: applications to the study of blood flow and jets by imaging techniques. J Am Coll Cardiol 1988;12:1344-1353.

6. Jacobs LE, Kotler MN, Parry WR. Flow patterns in dilated cardiomyopathy: a pulsedwave and color flow Doppler study. J Am Soc Echocardiogr 1990 3:294-302.

7. Hong GR, Pedrizzetti G, Tonti G, et al. Characterization and quantification of vortex flow in the human left ventricle by contrast echocardiography using vector particle image velocimetry. JACC Cardiovasc Imaging 2008 1:705-717.

8. Doenst T, Spiegel K, Reik M, et al. Fluid-dynamic modeling of the human left ventricle: methodology and application to surgical ventricular reconstruction. Ann Thorac Surg 2009 87:1187-1195.

9. Mohiaddin RH. Flow patterns in the dilated ischemic left ventricle studied by MR imaging with velocity vector mapping. J Magn Reson Imaging 1995;5:493-498. 
10. Eriksson J, Dyverfeldt P, Engvall J, Bolger AF, Ebbers T, Carlhäll CJ. Quantification of presystolic blood flow organization and energetics in the human left ventricle. Am J Physiol Heart Circ Physiol 2011;300:H2135-2141.

11. Eriksson J, Bolger AF, Ebbers T, Carlhäll CJ. Four-dimensional blood flow-specific markers of LV dysfunction in dilated cardiomyopathy. Eur Heart J Cardiovasc Imaging 2012;14:417-424.

12. Dyverfeldt P, Kvitting JPE, Sigfridsson A, Engvall J, Bolger AF, Ebbers T. Assessment of Fluctuating Velocities in Disturbed Cardiovascular Blood Flow: In Vivo Feasibility of Generalized Phase-Contrast MRI. J Magn Reson Imaging 2008;28:655-663.

13. Dyverfeldt P, Kvitting JPE, Carlhäll CJ, et al. Hemodynamic Aspects of Mitral Regurgitation Assessed by Generalized Phase-Contrast MRI. J Magn Reson Imaging 2011;33:582-588.

14. Dyverfeldt P, Sigfridsson A, Kvitting JPE, Ebbers T. Quantification of Intravoxel Velocity Standard Deviation and Turbulence Intensity by Generalizing Phase-Contrast MRI. Magn Reson Med 2006;56:850-858.

15. Dyverfeldt P, Hope MD, Tseng EE, Saloner D. Magnetic resonance measurement of turbulent kinetic energy for the estimation of irreversible pressure loss in aortic stenosis. JACC Cardiovasc Imaging 2013;6:64-71.

16. Binter C, Knobloch V, Manka R, Sigfridsson A, Kozerke S. Bayesian multipoint velocity encoding for concurrent flow and turbulence mapping. Magn Reson Med 2013;69:1337-1345.

17. Stein PD, Sabbah HN. Turbulent blood flow in the ascending aorta of humans with normal and diseased aortic valves. Circ Res 1976;39:58-65. 
18. Reynolds O. An Experimental Investigation of the Circumstances Which Determine Whether the Motion of Water Shall Be Direct or Sinuous, and of the Law of Resistance in Parallel Channels. Phil Trans R Soc Lond 1883;174:935-982.

19. Eriksson J, Carlhäll CJ, Dyverfeldt P, Engvall J, Bolger AF, Ebbers T. Semi-automatic quantification of 4D left ventricular blood flow. J Cardiovasc Magn Reson 2010;12.

20. Mathieu J, Scott J. An introduction to turbulent flow. Cambridge: Cambridge University Press; 2000.

21. Heiberg E, Sjögren J, Ugander M, Carlsson M, Engblom H, Arheden H. Design and validation of Segment--freely available software for cardiovascular image analysis. BMC Med Imaging 2010;10.

22. Dyverfeldt P, Gårdhagen R, Sigfridsson A, Karlsson M, Ebbers T. On MRI Turbulence Quantification. Magnetic resonance imaging 2009;27:913-922.

23. Carlhäll CJ, Bolger A. Passing strange: flow in the failing ventricle. Circ Heart Fail 2010;3:326-331.

24. Knobloch V, Binter C, Gülan U, et al. Mapping mean and fluctuating velocities by Bayesian multipoint MR velocity encoding-validation against 3D particle tracking velocimetry. Magn Reson Med 2013:In Press.

25. Sigfridsson A, Petersson S, Carlhäll C, Ebbers T. Four-dimensional flow MRI using spiral acquisition. Magn Reson Med 2012;68:1065-1073. 


\section{TABLES}

Table 1: Demographical and clinical data for the study population.

\begin{tabular}{lccc}
\hline & $\begin{array}{c}\text { DCM patients } \\
(\mathbf{n}=9)\end{array}$ & $\begin{array}{c}\text { Healthy subjects } \\
(\mathbf{n}=\mathbf{1 1})\end{array}$ & P-value \\
\hline Gender (male:female) & $4: 5$ & $6: 5$ & - \\
Age (yrs) & $51 \pm 13$ & $43 \pm 18$ & 0.30 \\
Weight (kg) & $77 \pm 13$ & $72 \pm 8$ & 0.26 \\
Heart Rate (bpm) & $62 \pm 12$ & $71 \pm 10$ & 0.08 \\
BP Systolic (mmHg) & $122 \pm 15$ & $127 \pm 12$ & 0.40 \\
BP Diastolic (mmHg) & $77 \pm 10$ & $77 \pm 7$ & 0.99 \\
LVEF (\%) & $41 \pm 5$ & $62 \pm 3$ & $<0.001$ \\
LVEDV (ml) & $173 \pm 33$ & $144 \pm 25$ & $<0.05$ \\
LV diastolic function according to echo Doppler indices & \\
Normal & 4 & 11 & - \\
Relaxation abnormality & 2 & - & - \\
Pseudonormal filling & 1 & - & - \\
\hline
\end{tabular}

Mean \pm SD; bpm, beats per minute; BP, Blood Pressure 
Table 2: LV inflow characteristics at the time of transmitral peak velocity during early $(\mathrm{E})$ and late (A) diastolic filling.

\section{DCM patients Healthy subjects P-value}

\begin{tabular}{lccc}
\hline LV diameter E (cm) & $5.4 \pm 0.8$ & $3.7 \pm 0.4$ & $<0,001$ \\
LV diameter A (cm) & $6.0 \pm 0.6$ & $4.3 \pm 0.5$ & $<0,001$ \\
Mitral annular diameter E (cm) & $3.3 \pm 0.6$ & $3.1 \pm 0.3$ & 0.35 \\
Mitral annular diameter A (cm) & $3.3 \pm 0.7$ & $2.9 \pm 0.3$ & 0.09 \\
Transmitral peak velocity E (m/s) & $0.81 \pm 0.1$ & $0.94 \pm 0.2$ & 0.09 \\
Transmitral peak velocity A (m/s) & $0.71 \pm 0.3$ & $0.61 \pm 0.1$ & 0.31 \\
\hline
\end{tabular}

Mean \pm SD 


\section{FIGURES}

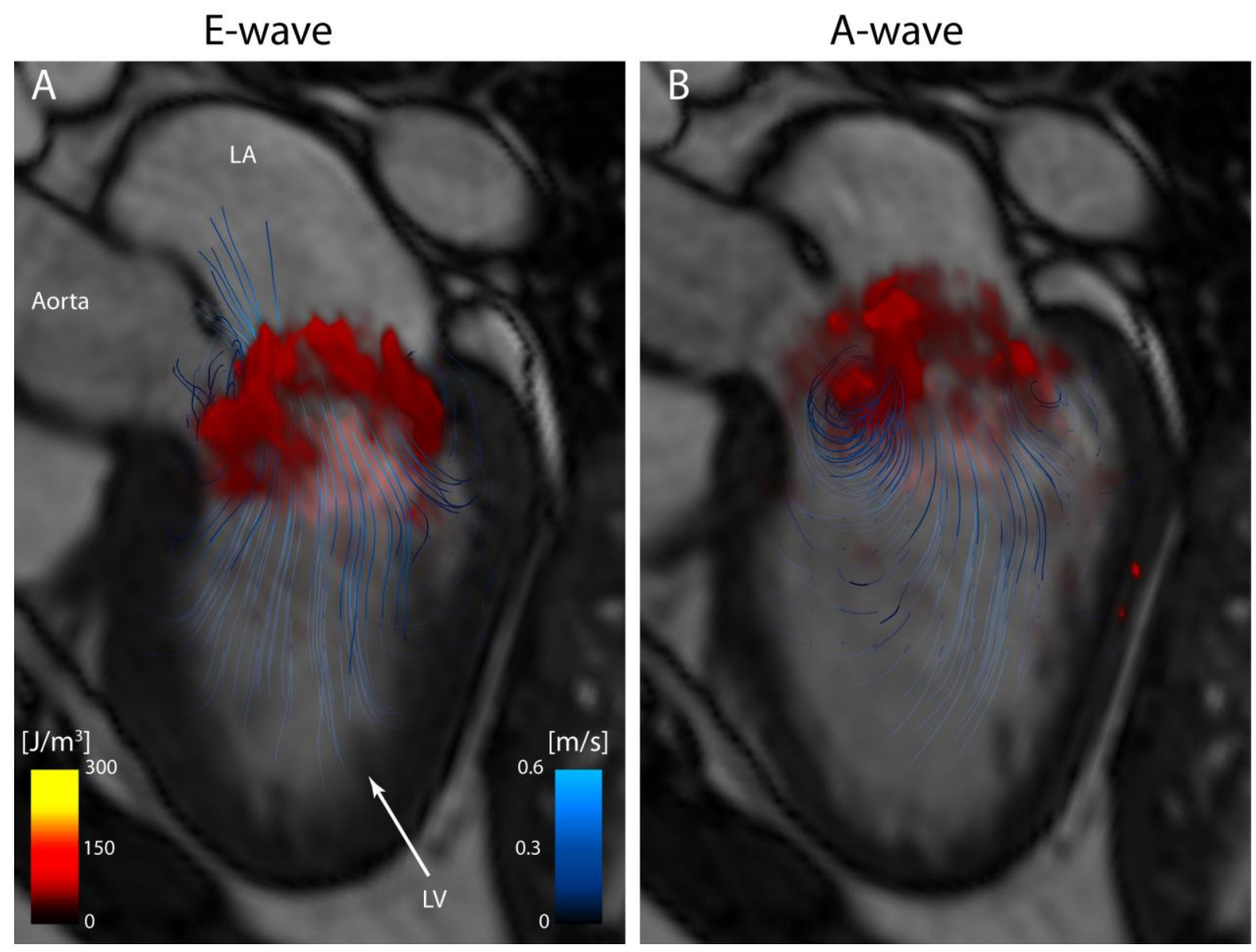

Figure 1: 3D volume rendering of TKE (red) and streamlines (blue) in a normal LV at early (left) and late (right) diastolic filling. A semitransparent grayscale three-chamber image, tilted approximately $30^{\circ}$ from a vertical plane, provides morphological orientation. 


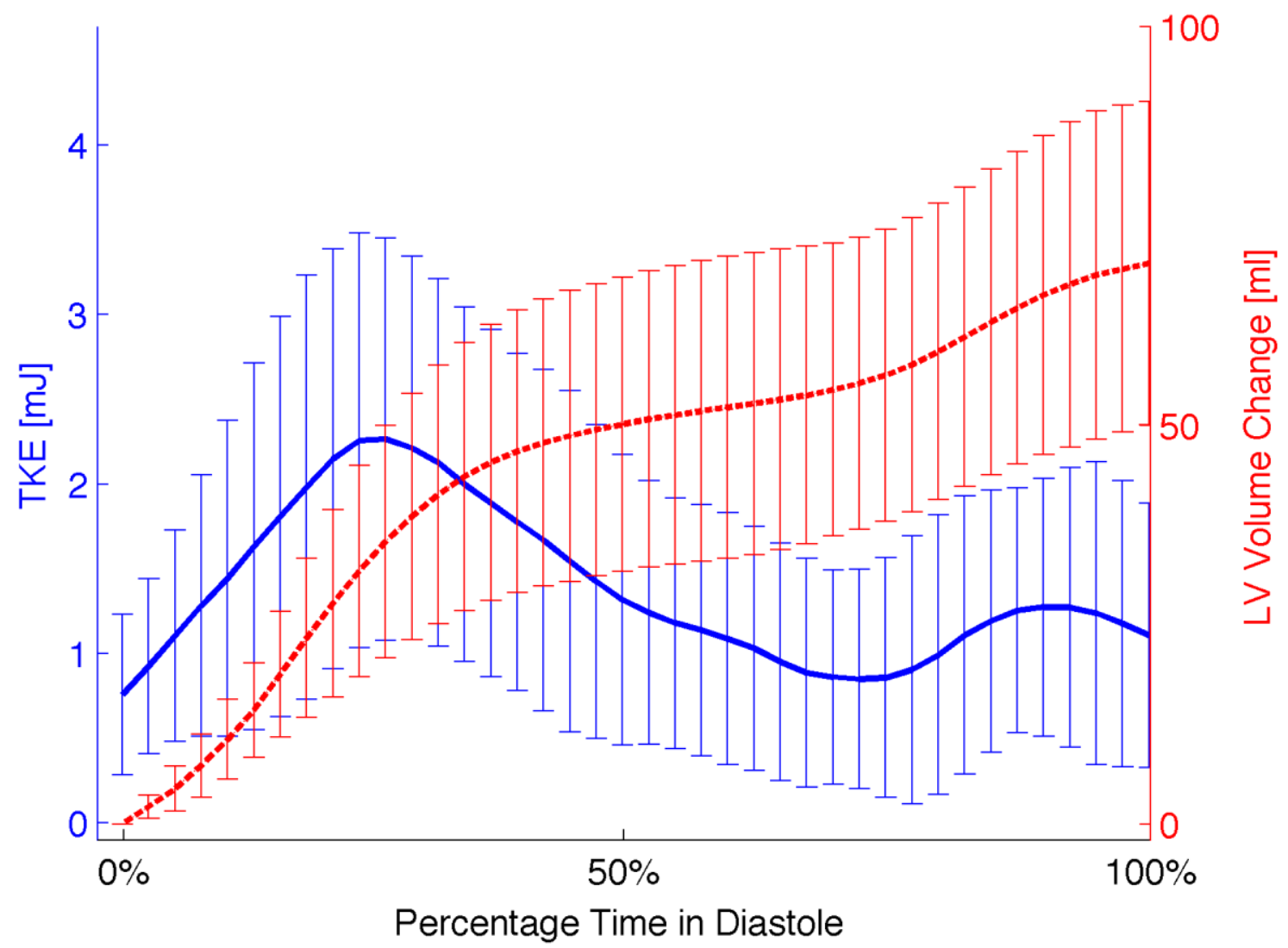

Figure 2: TKELV (blue solid line) and LV volume change (red dashed line) over diastole (mean \pm SD) for healthy subjects $(n=11)$. 

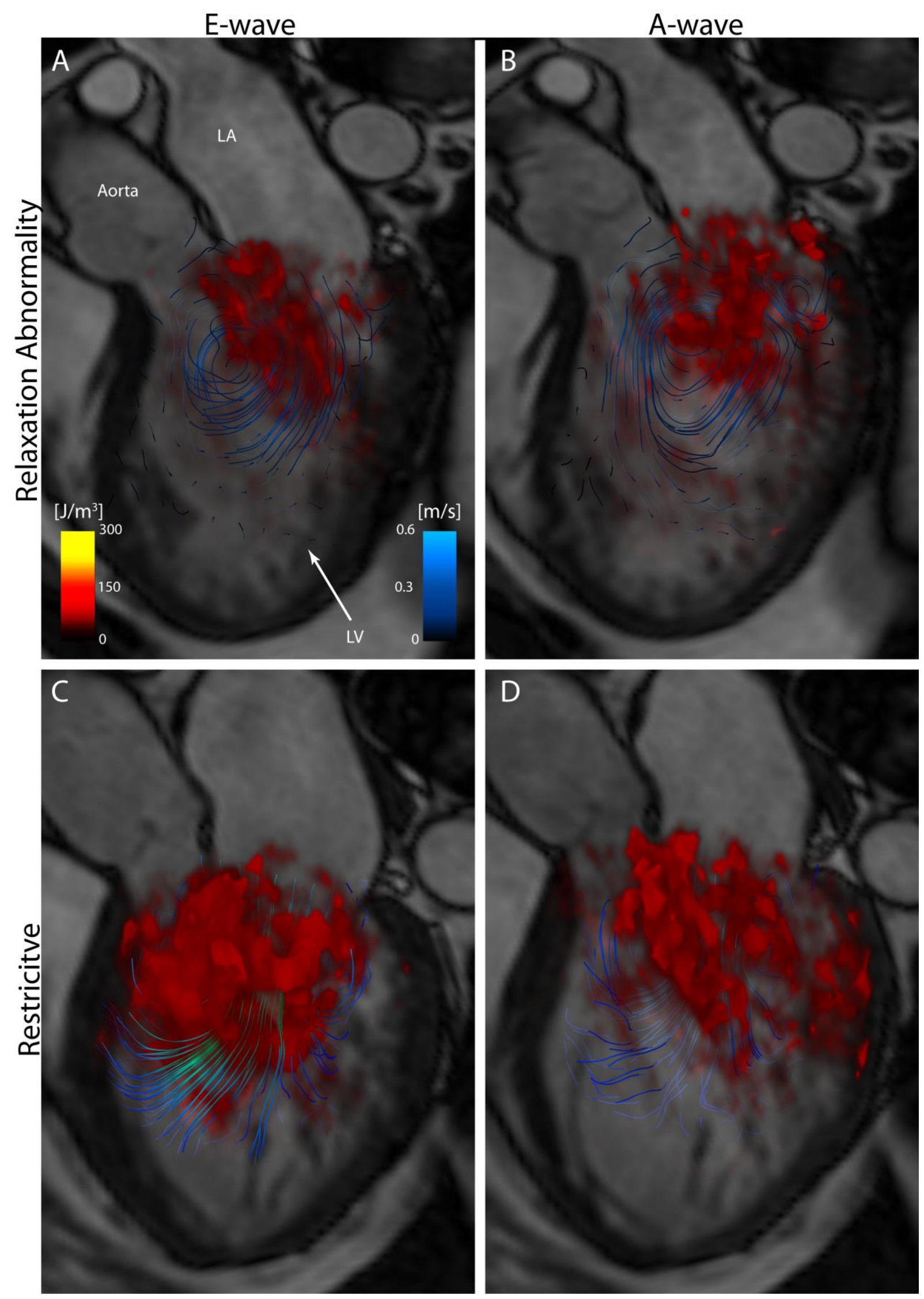

Figure 3: 3D volume rendering of TKE (red) and streamlines (blue) in DCM patients at early (left) and late (right) diastolic filling. Top panels, a patient with relaxation abnormality (mild 
LV diastolic dysfunction); and bottom panels, a patient with restrictive filling (severe LV diastolic dysfunction). A semitransparent grayscale three-chamber image, tilted approximately $30^{\circ}$ from a vertical plane, provides morphological orientation.
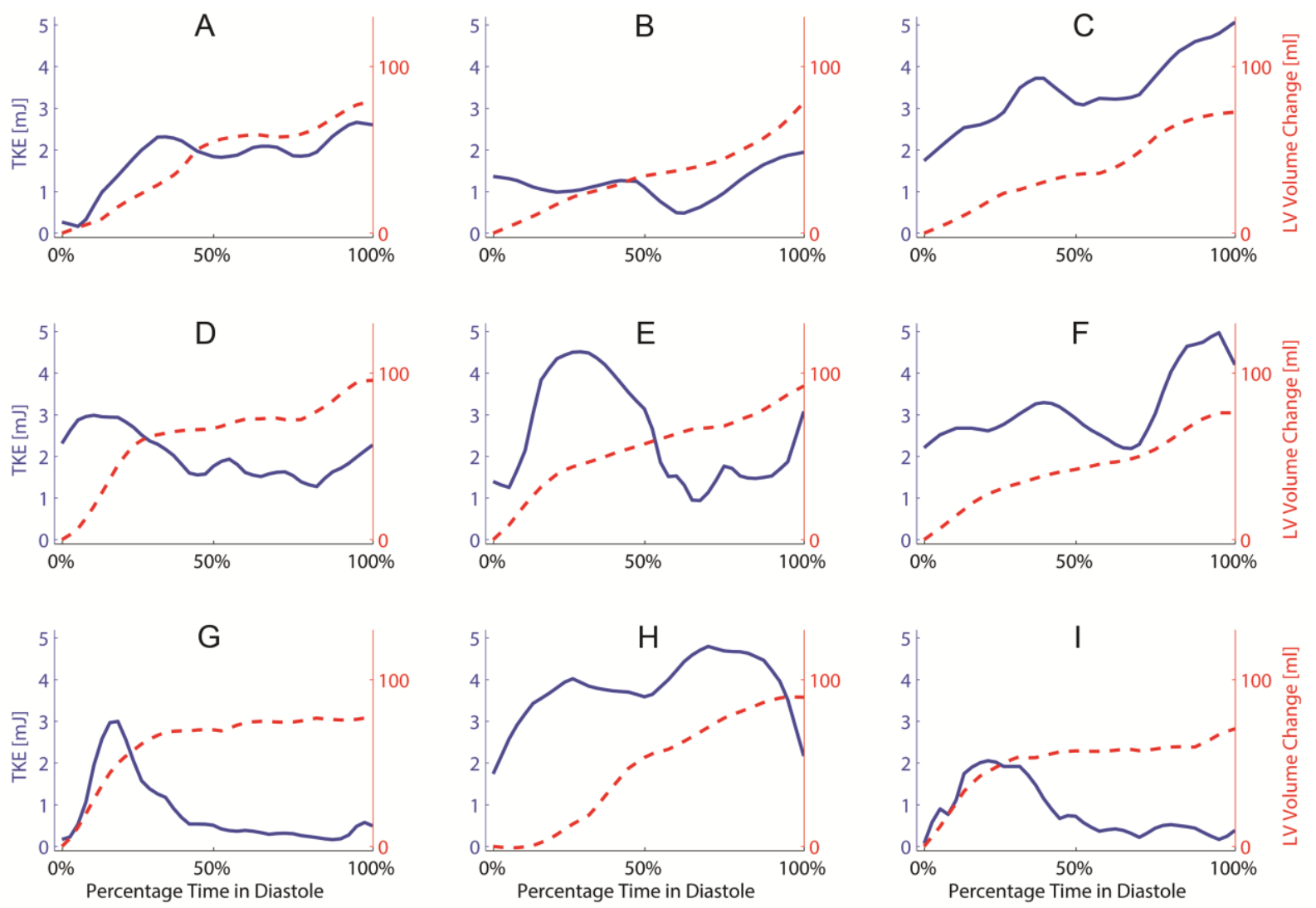

Figure 4: TKE $L$ (blue solid lines) and LV volume change (red dashed lines) over diastole in a heterogeneous group of DCM patients with different degrees of diastolic dysfunction.

According to conventional echo Doppler indices at rest, four patients had normal diastolic function (A, C, H and I), two had relaxation abnormality (B and F), one had pseudonormal filling (D) and two had restrictive filling (E and G). 


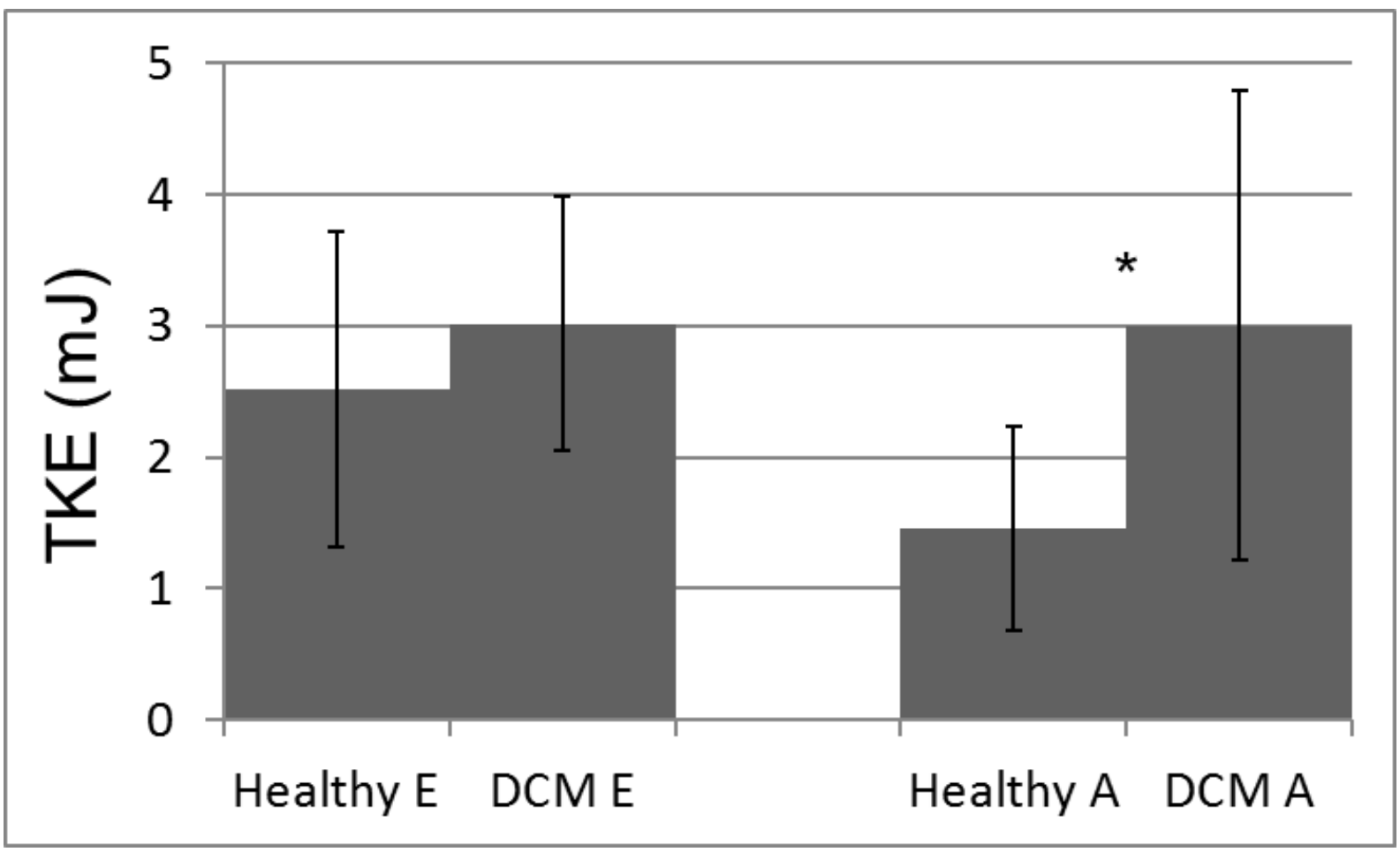

Figure 5: Bar graph illustrating peak TKELV at early (E) and late (A) diastolic filling in healthy subjects and DCM patients. ${ }^{*} \mathrm{p}=0.02$ (DCM vs Healthy at A) 
$E$
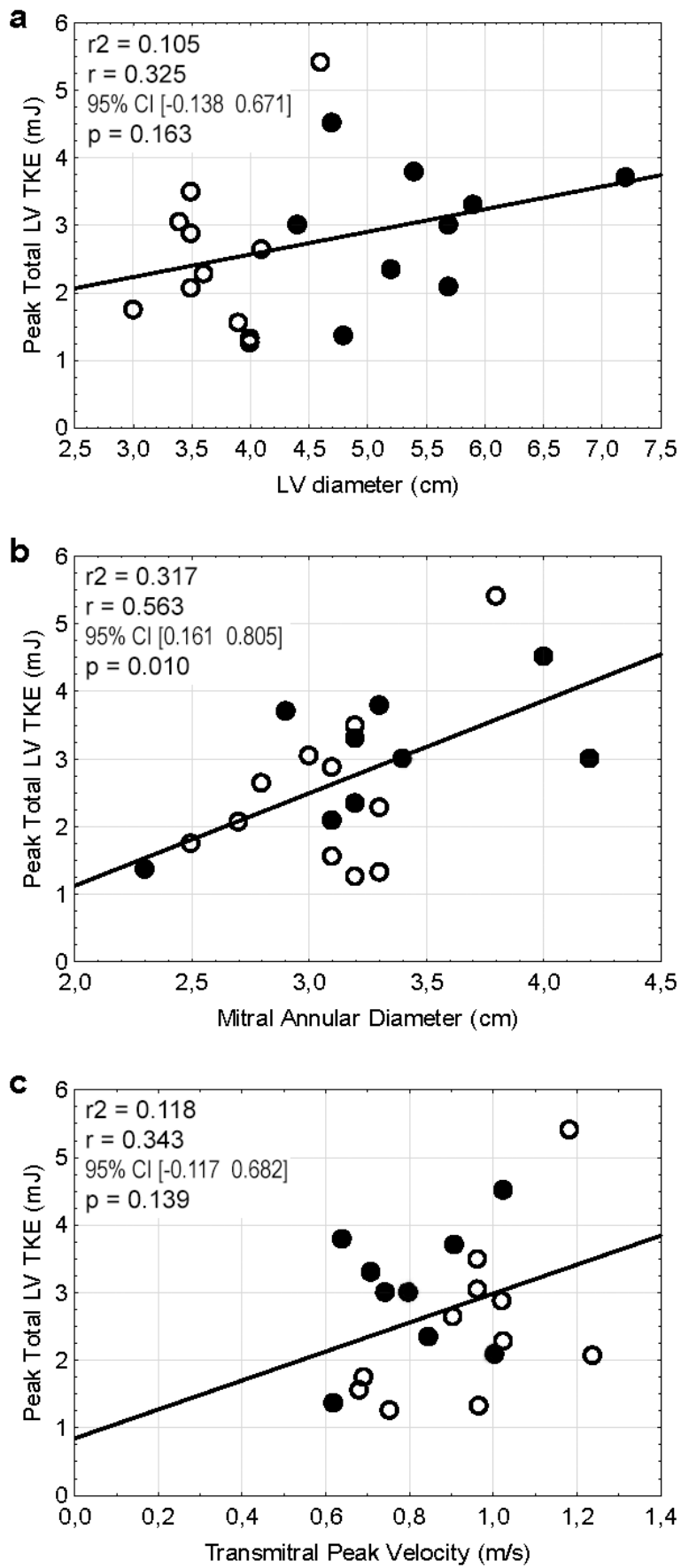

A
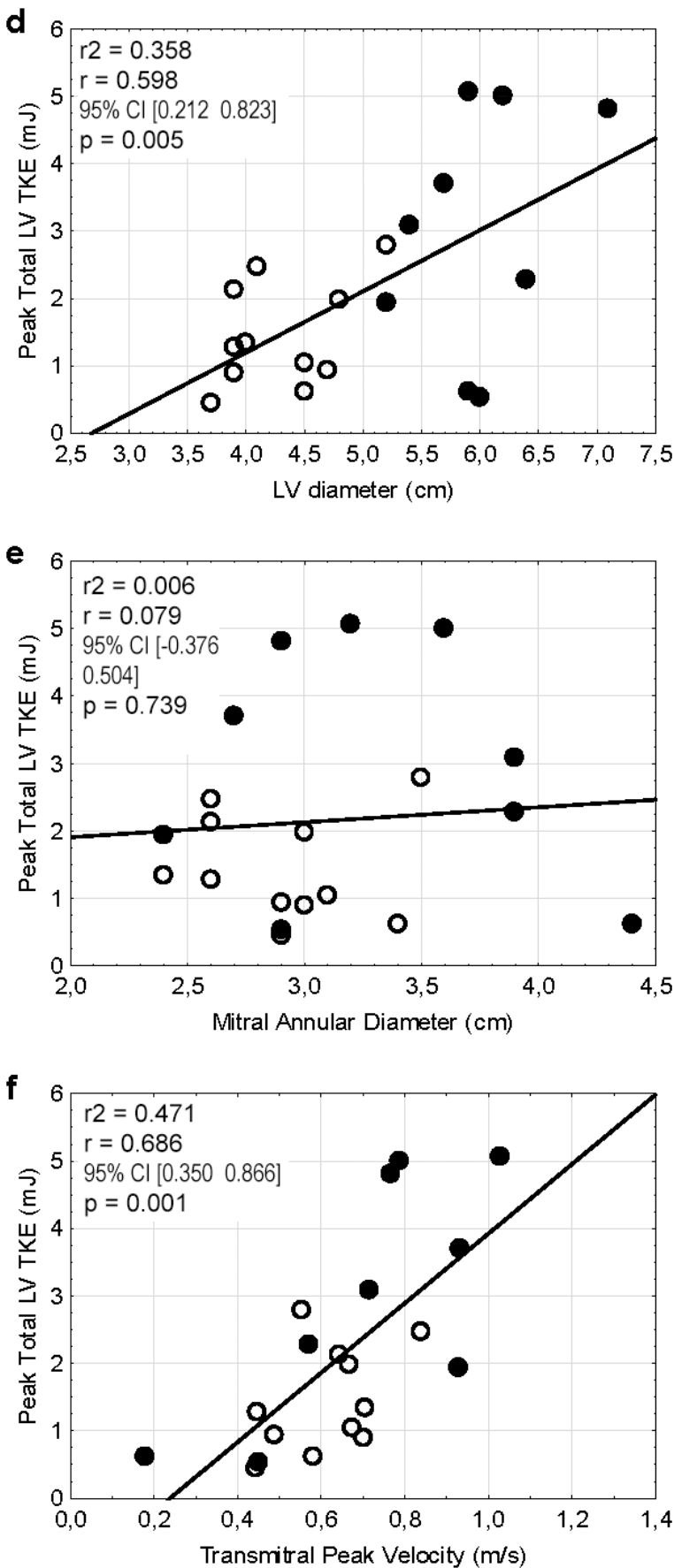

Figure 6 (a-f): Correlation between peak TKELV and LV diameter, mitral annular diameter, and transmitral peak velocity during early (E) (left panels) and late (A) (right panels) diastolic filling. $\bigcirc=$ healthy subjects,$\bullet=$ DCM patients. $\mathrm{CI}=$ confidence interval . 


\section{MOVIE LEGENDS}

Movie S1: 3D volume rendering of turbulent kinetic energy (red) and streamlines (blue) visualized throughout diastole in a left ventricle of a healthy subject. A semitransparent grayscale three-chamber image, tilted approximately $30^{\circ}$ from a vertical plane, provides morphological orientation.

Movie S2: 3D volume rendering of turbulent kinetic energy (red) and streamlines (blue) visualized throughout diastole in a left ventricle of a patient with relaxation abnormality (mild LV diastolic dysfunction). A semitransparent grayscale three-chamber image, tilted approximately $30^{\circ}$ from a vertical plane, provides morphological orientation.

Movie S3: 3D volume rendering of turbulent kinetic energy (red) and streamlines (blue) visualized throughout diastole in a left ventricle of a patient with restrictive filling (severe LV diastolic dysfunction). A semitransparent grayscale three-chamber image, tilted approximately $30^{\circ}$ from a vertical plane, provides morphological orientation. 